\title{
ASYMPTOTIC ESTIMATES OF THE $n$-WIDTHS IN HILBERT SPACE
}

\author{
JOSEPH W. JEROME ${ }^{1}$
}

\begin{abstract}
Let $\Omega \subset R^{m}$ be a bounded open set satisfying the restricted cone property and let $R$ be a nonnegative selfadjoint operator on $L^{2}(\Omega)$ which is the realization of a uniformly elliptic operator $A$ of order $v$ with suitable coefficients and principal part $a(x, \xi)$. Let $\mathscr{R}$ be the ellipsoid $\{f:(R f, f) \leqq 1\}$. The $L^{2}$ in-widths $d_{n}(\mathscr{R})$ satisfy $d_{n}(\mathscr{R}) \sim(c / n)^{v / 2 m}$ where $c=\int_{\Omega}\left(\int_{0<a(x, \xi)<1} d \xi\right) d x$. If $B(u, v)$ is a nonnegative Hermitian coercive form ove: a subspace $\boldsymbol{V}^{\gamma}$ of the Sobolev space $W^{i .2}(\Omega)$, then the $n$-widths of $: B=\left\{f \in Y^{\prime}: B(f, f) \leqq 1\right\}$ satisfy, $0<\left(c^{\prime}\right)^{k / m} \leqq \lim$ inf $d_{n}(\mathscr{B}) n^{k / m} \leqq$ lim sup $d_{n}(\not B) n^{i / m} \leqq\left(c^{\prime \prime}\right)^{k / m}$. In some cases $c^{\prime}=c=c^{\prime \prime}$ where $c$ is defined in terms of an elliptic operator of order $2 k$. The $n$-widths of $\mathscr{B}$ in $W^{j, 2}(\Omega), 0 \leqq j \leqq k$, are of order $O\left(n^{-(k-j) / m}\right), n \rightarrow \infty$.
\end{abstract}

The purpose of this note is to supplement and strengthen certain of the results contained in [6], [7] and [8] through the use of results of Beals ([2], [3], [4]) on the asymptotic distribution of eigenvalues of elliptic operators. The relationship between Hilbert space $n$-widths of classes, satisfying quadratic inequalities, and the eigenvalues of the associated selfadjoint linear operators was described in the above references ([6], [7], [8]). The $n$-widths are characterized, roughly speaking, as the square roots of the reciprocals of the positive eigenvalues arranged in increasing order according to multiplicity. This characterization facilitates the calculation of asymptotic estimates for $n$-widths when such estimates are available for eigenvalues. In particular, we obtain such estimates in this paper, with precise constants in certain instances, for a broader class of Euclidean domains and also for more representative function classes than had been obtained previously. In this connection, we appreciably weaken the assumptions on the smoothness of the coefficients of the underlying elliptic operators.

Let $\Omega \subset R^{m}$ be a bounded open set satisfying the restricted cone property $\left[1\right.$, p. 11] and let $L^{2}(\Omega)$ and $W^{k, 2}(\Omega)$ denote the complex Lebesgue and Sobolev Hilbert spaces with standard inner product $[1$, p. 4]. Let $R$ be

Received by the editors October 4, 1971.

AMS 1970 subject classifications. Primary 41 A25, 41 A45; Secondary 35P15, 35P20.

Key words and phrases. n-widths, asymptotic distribution, eigenvalues, elliptic, quadratic inequalities, selfadjoint, coercive, restricted cone condition, Sobolev spaces.

${ }^{1}$ Work supported by National Science Foundation grant GP-19526. 
a selfadjoint, nonnegative linear operator with domain $\mathscr{D}_{R} \subset L^{2}(\Omega)$ which is the realization of a formally selfadjoint uniformly elliptic [1, p. 45] operator,

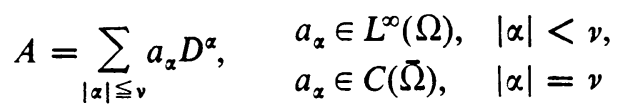

on $\Omega$, such that $\mathscr{D}_{R} \subset W^{v, 2}(\Omega)$. By the term realization here is meant that $R$ is a restriction of $A$ such that $\mathscr{D}_{R} \supset C_{0}^{\infty}(\Omega)$, the infinitely differentiable functions with compact support on $\Omega$, where $A$ is defined on an appropriately smooth function class in $W^{v, 2}(\Omega)$. By the ellipsoid $\mathscr{R}$ determined by $R$ we mean the class

$$
\mathscr{R}=\left\{f \in \mathscr{D}_{R}:(R f, f)_{L^{2}(\Omega)} \leqq 1\right\}
$$

and by the $L^{2}(\Omega) n$-width of $\mathscr{R}, n \geqq 0$, we mean the number $d_{n}(\mathscr{R})$,

$$
d_{n}(\mathscr{R})=\inf _{\operatorname{dim} \mathscr{H}=n} E(\mathscr{R}, \mathscr{M}),
$$

$$
E(\mathscr{R}, \mathscr{M})=\sup _{f \in \mathscr{R}} \inf _{g \in_{\mathcal{M}}}\|f-g\|_{L^{2}(\Omega)} .
$$

THEOREM 1. The (extended real) numbers $d_{n}(\mathscr{R})$ satisfy the asymptotic relationship

$$
d_{n}(\mathscr{R})=(c / n)^{\mathrm{v} / 2 m}+o\left(n^{-v / 2 m}\right), \quad n \rightarrow \infty,
$$

where

$$
\begin{aligned}
c & =(2 \pi)^{-m} \int_{\Omega} w(x) d x, \\
w(x) & =\operatorname{measure}\left\{\xi: 0<a(x, \xi)=\sum_{|x|=v} a_{x}(x) \xi^{\alpha}<1\right\} .
\end{aligned}
$$

Proof. Since $\mathscr{D}_{R} \subset W^{v, 2}(\Omega)$ and the injection $W^{v, 2}(\Omega) \rightarrow L^{2}(\Omega)$ is compact [1, pp. 30,11], the spectral representation of the selfadjoint, nonnegative linear operator $R$ may be written $R=\sum_{i} \lambda_{i} P_{i}$ where $0 \leqq \lambda_{1} \leqq$ $\lambda_{2} \leqq \cdots$ are eigenvalues of finite multiplicity and $P_{1}, P_{2}, \cdots$ are projectors onto one-dimensional subspaces of $L^{2}(\Omega)$. Then by Theorem 2.1 of [6] we have

$$
d_{n}(\mathscr{R})=\hat{\lambda}_{n+1}^{-1 / 2}, \quad n=0,1, \cdots .
$$

It is understood here that $d_{n}(\mathscr{R})=\infty$ for each $n$ such that $\lambda_{n+1}=0$. By the theorem of [4], the asymptotic distribution of the above eigenvalues satisfies the relationship

$$
N(\hat{\lambda})=c \lambda^{m / v}+o\left(\lambda^{m / v}\right), \quad \lambda \rightarrow \infty,
$$


where $c$ is given by (5) and $N(\lambda)$ denotes the number of eigenvalues less than or equal to $\lambda_{\text {. Since }} N\left(\hat{\lambda}_{j+1}\right)=N\left(\lambda_{j}\right)(1+o(1)$ ) (cf. the proof of Lemma 3.2 of [8]) a routine argument shows that (7) implies

$$
\lambda_{j}=(j / c)^{v / m}+o\left(j^{v / m}\right), \quad j \rightarrow \infty .
$$

(8) and (6) taken together imply (4) and the proof is completed.

For $k \geqq 1$, let $\mathscr{V}^{\circ}$ be a complete space satisfying

$$
W_{0}^{k, 2}(\Omega) \subset \mathscr{V} \subset W^{k, 2}(\Omega)
$$

where $W_{0}^{k, 2}$ is the completion in $W^{k, 2}(\Omega)$ of $C_{0}^{\infty}(\Omega)$. Let $B(f, g)$ be a nonnegative Hermitian form on $\mathscr{V}$ defined by

$$
B(f, g)=\sum_{|\alpha| \leqq k ;|\beta| \leqq k} \int_{\Omega} a_{\alpha \beta} D^{\alpha} f \cdot D^{\beta} \bar{g}
$$

where $a_{\alpha, \beta} \in C(\bar{\Omega}) \cap W^{k, \infty}(\Omega)$ if $|\alpha|+|\beta|=2 k$ and $a_{a, \beta} \in W^{|\alpha| \cdot \infty}$ if $|\alpha|+$ $|\beta|<2 k$. Here, $W^{v, \infty}(\Omega)$ denotes the Sobolev class of complex functions $f$ such that $D^{\alpha} f \in L^{\infty}(\Omega)$ for $|\alpha| \leqq v$, where differentiation is taken in the sense of distributions. We retain the assumption of uniform ellipticity on the associated operator $A$ of (12) below and we assume that $B(f, f)$ is coercive, i.e., for all $f \in \mathscr{V}$,

$$
B(f, f) \geqq c_{0}(f, f)_{W^{k, 2}(\Omega)}-\mu_{0}(f, f)_{L^{2}(\Omega)},
$$

with $c_{0}>0, \mu_{0} \geqq 0$. In this case, by Theorem 2.1 of [8] there exists a unique selfadjoint operator $R$ on $L^{2}(\Omega)$ with $C_{0}^{\infty}(\Omega) \subset \mathscr{D}_{R} \subset \mathscr{V}$ satisfying

$$
B(f, g)=(R f, g)_{L^{2}(\Omega)} \text { for all } f \in \mathscr{D}_{R}, g \in \mathscr{V},
$$

with $\mathscr{D}_{R}$ characterized by (11) and dense in $\mathscr{V}$ in the topology of $W^{k, 2}(\Omega)$. In particular, $R=A$ on the class $C_{0}^{\infty}(\Omega)$ where

$$
A f=\sum_{|\alpha| \leqq k ;|\beta| \leqq k}(-1)^{|\alpha|} D^{\alpha}\left[a_{\alpha \beta} D^{\beta} f\right],
$$

and $R$ has a spectral representation $R=\sum_{j=1}^{\infty} \lambda_{j} P_{j}$. Define now the class $\mathscr{B}=\{f \in \mathscr{V}: B(f, f) \leqq 1\}$ and denote by $d_{n}^{(j)}(\mathscr{B})$ the $n$-widths of $\mathscr{B}$ in $W^{j .2}(\Omega), 0 \leqq j \leqq k$. Here $d_{n}^{(0)}(\mathscr{B})=d_{n}(\mathscr{B})$.

THEOREM 2. Let $c$ be given by (5) with $a(x, \xi)=\sum_{|\alpha|+|\beta|=2 k} a_{\alpha \beta}(x) \xi^{\alpha+\beta}$. $\Omega$ is a bounded open set satisfying the restricted cone condition unless further restricted. Then,

(13i) if $\Omega$ is a domain with $C^{\infty}$ boundary,

$$
d_{n}\left(\mathscr{B} \cap W_{0}^{k, 2}(\Omega)\right)=(c / n)^{k / m}+o\left(n^{-k / m}\right), \quad n \rightarrow \infty ;
$$


(13ii) there exist positive constants $c^{\prime} \leqq c \leqq c^{\prime \prime}$ such that

$$
\left(c^{\prime}\right)^{k / m} \leqq \liminf _{n \rightarrow \infty} d_{n}(\mathscr{B}) n^{k / m} \leqq \limsup _{n \rightarrow \infty} d_{n}(\mathscr{B}) n^{k / m} \leqq\left(c^{\prime \prime}\right)^{k / m}
$$

(13iii) $d_{n}^{(j)}(\mathscr{B})=O\left(n^{-(k-j) / m}\right), 0 \leqq j \leqq k$;

(13iv) if $\mathscr{D}_{R} \subset W^{2^{k, 2}}(\Omega)$ and $R=A$ on $\mathscr{D}_{R}$, where $R$ is defined by (11),

$$
d_{n}(\mathscr{B})=(c / n)^{k / m}+o\left(n^{-k / m}\right), \quad n \rightarrow \infty \text {. }
$$

Proof. (13i) Under the assumption of (i), $\Omega$ is a $C^{\infty}$ Riemannian manifold with boundary. According to [2, p. 703] the operator $A$ defined on $W^{2 k, 2}(\Omega) \cap W_{0}^{k, 2}(\Omega)$ by (12) is selfadjoint. Therefore, Theorem 1 with $\mathscr{V}=W_{0}^{k, 2}(\Omega)$ and $A=R$ implies (13i). By way of remark, it is easily seen that $A$ coincides in this case with the operator $R$ constructed by means of (11). In the proofs of (13ii), (13iii) and (13iv) to follow, $R$ will denote the operator of (11) and $\lambda_{j}$ its eigenvalues.

(13ii) By Theorems 1.4 and 4.1 of [3] the eigenvalues $\mu_{1} \geqq \mu_{2} \geqq \cdots>0$ of $S=\left(R+\mu_{0} I\right)^{-1 / 2}$ satisfy the property that the sequence $\mu_{j} j^{k / m}$ is bounded; indeed the only technical verification required is that the range of $S$ is contained in $W^{k, 2}(\Omega)$, a fact which follows from the identities

$$
\mathscr{D}_{S^{-1}}=\left\{u \in L^{2}: \sum_{j} \mu_{j}^{-2}\left|\left(u, \varphi_{j}\right)\right|_{L^{2}(\Omega)}^{2}<\infty\right\}=\mathscr{V} .
$$

Here the $\left\{p_{j}\right\}$ are a complete orthonormal system in $L^{2}(\Omega)$ and a complete orthogonal system in $\mathscr{V}$ satisfying $S_{\varphi_{j}}=\mu_{j} \varphi_{j}$. Thus, the eigenvalues $\lambda_{j}$ of $R$ satisfy $\left(\lambda_{j}+\mu_{0}\right)^{-1 / 2} \leqq c_{*} J^{-k / m}$ for some $c_{*}>0$, for all $j$. Equation (6) and simple computations then yield

$$
\limsup _{n \rightarrow \infty} d_{n}(\mathscr{R}) n^{k / m} \leqq\left(c^{\prime \prime}\right)^{k / m}
$$

for some $c^{\prime \prime}>0$, where $\mathscr{R}$ is defined by (2). Now $\mathscr{R}$ and $\mathscr{B}$ have identical closures in $L^{2}(\Omega)$ [6, Theorem 3.1] and hence identical $n$-widths. This verifies the second inequality of (13ii). To obtain the first inequality, let $\Omega^{\prime}$ be a domain with $C^{\infty}$ boundary satisfying $\Omega^{\prime} \subset \Omega$. Define $c^{\prime}$ by (5) with $\Omega$ replaced by $\Omega^{\prime}$ and $a(x, \xi)=\sum_{|\alpha|+|\beta|=2 k} a_{\alpha \beta}(x) \xi^{\alpha+\beta}$. One sees easily that $W_{0}^{k, 2}\left(\Omega^{\prime}\right) \subset W_{0}^{k, 2}(\Omega)$; if $u \in W_{0}^{k, 2}\left(\Omega^{\prime}\right)$, define $u(x)$ for $x \in \Omega-\Omega^{\prime}$ by $u(x)=0$. The extended function is the limit in $W_{0}^{k, 2}\left(\Omega^{\prime}\right)$ of $C_{0}^{\infty}\left(\Omega^{\prime}\right)$ functions. We then have

$$
\liminf _{n \rightarrow \infty} d_{n}(\mathscr{B}) n^{k / m} \geqq \liminf _{n \rightarrow \infty} d_{n}\left(\mathscr{B} \cap W_{0}^{k .2}\left(\Omega^{\prime}\right)\right) n^{k / m}=\left(c^{\prime}\right)^{k / m},
$$

with the latter equality a consequence of (13i).

(13iii) The result for $j=0$ follows from (13ii); for $j=k$ the result is a consequence of the following inequalities:

$$
d_{n}^{(k)}(\mathscr{B})=d_{n}^{(k)}(\mathscr{R}) \leqq c_{0}^{-1 / 2} \sup _{f \in \mathscr{R}}\left\{\sum_{v=n+1}^{\infty}\left|B\left(f, \psi_{v}\right)+\mu_{0}\left(f, \psi_{v}\right)_{L^{2}(\Omega)}\right|^{2}\right\}^{1 / 2},
$$


where $\psi_{v}=\varphi_{v} /\left(\lambda_{v}+\mu_{0}\right)^{1 / 2}$

$$
\leqq\left(2 / c_{0}\right)^{1 / 2} \sup _{f \in \mathscr{R}}\left\{\sum_{v=1}^{\infty}\left|B\left(f, \psi_{v}\right)\right|^{2}+\mu_{0}^{2} \sum_{v=n+1}^{\infty} \lambda_{v}^{-2}\left|B\left(f, \psi_{v}\right)\right|^{2}\right\}^{1 / 2},
$$

where $n$ is chosen sufficiently large so that $\lambda_{n+1}>0$,

$$
\begin{aligned}
& \leqq\left[\frac{2\left(1+\mu_{0}^{2} / \lambda_{n+1}^{2}\right)}{c_{0}}\right]^{1 / 2} \sup _{f \in \mathscr{R}}\left\{\sum_{v=1}^{\infty}\left|B\left(f, \psi_{v}\right)\right|^{2}\right\}^{1 / 2} \\
& =\left[\frac{2\left(1+\mu_{0}^{2} / \lambda_{n+1}^{2}\right)}{c_{0}}\right]^{1 / 2} \sup _{f \in \mathscr{R}}\left\{\sum_{v=1}^{\infty} \frac{\lambda_{v}}{\lambda_{v}+\mu_{0}}\left(R f,\left(f, \varphi_{v}\right)_{L^{2}(\Omega)} \varphi_{v}\right)_{L^{2}(\Omega)}\right\}^{1 / 2} \\
& \leqq\left[\frac{2\left(1+\mu_{0}^{2} / \lambda_{n+1}^{2}\right)}{c_{0}}\right]^{1 / 2} \sup _{f \in \mathscr{H}}\left\{(R f, f)_{L^{2}(\Omega)}\right\}^{1 / 2} .
\end{aligned}
$$

Thus, the result is established for $j=k$.

To obtain the result for $0<j<k$, we use the interpolation inequality [1, Lemma 13.3]

$$
\|u\|_{W^{j, 2}(\Omega)} \leqq \gamma\|u\|_{L^{2}(\Omega)}^{1-(j / k)}\|u\|_{W^{k, 2}(\Omega)}^{j / k}
$$

Now [8, Theorem 2.1]

$$
d_{n}(\mathscr{B})=E\left(\mathscr{B}, \operatorname{span}\left\{\varphi_{1}, \cdots, \varphi_{n}\right\}\right)
$$

and for any $f \in \mathscr{B}$, the best $L^{2}(\Omega)$ approximation element from $\operatorname{span}\left\{\varphi_{1}, \cdots, \varphi_{n}\right\}$ is $\sum_{v=1}^{n}\left(f, \varphi_{v}\right) \varphi_{v}=f_{n}$. Substituting $f-f_{n}$ for $u$ in (15) yields

$$
\left\|f-f_{n}\right\|_{W^{j, 2}(\Omega)} \leqq \gamma\left[d_{n}(\mathscr{R})\right]^{1-(j / k)}\left\|f-f_{n}\right\|_{W^{k, 2}(\Omega)}^{j / k}
$$

and the proof of the case $j=k$ shows that the numbers $\| f-f_{n} ! W^{k, 2(\Omega)}$ are bounded whenever $n \geqq n_{0}, \lambda_{n_{0}}>0$, independently of $f \in \mathscr{R}$ and hence $f \in \mathscr{B}$ [3, Theorem 3.1]. (17) and (13iii) for $j=0$ yield (13iii) for $0<j<k$.

(13iv) This is a consequence of Theorem 1 and the fact, already mentioned, that $d_{n}(\mathscr{R})=d_{n}(\mathscr{B})$.

REMARKS. (1) Theorem 2 (13ii) is a significant extension of Corollary (3.4) of [8]. In particular, the class of forms $B(u, v)$ and the class of domains $\Omega$ for which the theorem holds are both extended.

(2) Beals [5, Theorem 4] gives necessary and sufficient conditions that a strongly elliptic operator of order $2 k$, defined on a subspace of $W^{2 k .2}(\Omega)$, be selfadjoint and semibounded. In particular, these conditions give relevance to (13iv) of Theorem 2 .

(3) The proof of (13ii) shows that, if $\Omega$ has the property that it can be approximated from within by domains $\Omega_{\varepsilon}$, with $C^{\infty}$ boundaries, satisfying 
$\Omega_{\varepsilon} \subset \Omega$, measure $\left(\Omega-\Omega_{\varepsilon}\right)<\varepsilon$ for all $\varepsilon>0$, then $c^{\prime}$ in (2) can be chosen equal to $c$. Moreover, if $\Omega$ can be approximated from without in a similar manner, and the form $B(u, v)$ and its coefficients satisfy the stated hypotheses in a domain $\Omega^{\prime \prime} \supset \Omega$, then $c^{\prime \prime}$ may be taken equal to $c$ if $\mathscr{V}=W_{0}^{k, 2}(\Omega)$.

(4) (13iii) of Theorem 2 extends Theorem 3.6 of [8], whereas Theorem 1 and Theorem 2 (13iv) supplement the results of that reference. The results obtained in Theorems 1 and 2 depended on the characterization of $n$ widths in terms of eigenvalues and on asymptotic estimates for eigenvalues. It is also possible, as was done in $[7, \S 5]$, to use the characterization and estimates for $n$-widths to obtain estimates for eigenvalues.

(5) In [9], L. Schumaker and the writer by different methods obtained $L^{p}(\Omega) n$-width estimates, $\Omega$ an $m$-dimensional parallelopiped, for classes of smooth functions satisfying uniform norm constraints on derivatives up to a given order for $1 \leqq p \leqq \infty$. In the present work, the constraints are quadratic ones. The results suggest, however, that, in certain cases, classes of smooth functions satisfying quadratic constraints have the same closure in $L^{2}(\Omega)$ and in Sobolev space as classes of functions satisfying uniform norm constraints on their derivatives.

\section{REFERENCES}

1. S. Agmon, Lectures on elliptic boundary value problems, Van Nostrand Math. Studies, no. 2, Van Nostrand, Princeton, N.J., 1965. MR 31 \#2504.

2. R. Beals, On eigenvalue distributions for elliptic operators without smooth coefficients, Bull. Amer. Math. Soc. 72 (1966), 701-705. MR 33 \#4449.

3. - Classes of compact operators and eigenvalue distributions for elliptic operators, Amer. J. Math. 89 (1967), 1056-1072. MR 37 \#91.

4. - On eigenvalue distributions for elliptic operators without smooth coefficients. II, Bull. Amer. Math. Soc. 74 (1968), 1022-1024. MR 38 \#1414.

5. - Asymptotic behavior of the Green's function and spectral function of an elliptic operator, J. Functional Analysis 5 (1970), 484-503.

6. J. W. Jerome, On the $L_{2} n$-width of certain classes of functions of several variables, J. Math. Anal. Appl. 20 (1967), 110-123. MR 35 \#7060.

7. - Asymptotic estimates of the $L_{2} n$-width, J. Math. Anal. Appl. 22 (1968), 449-464. MR 37 \#4484.

8. - On n-widths in Sobolev spaces and applications to elliptic boundary value problems, J. Math. Anal. Appl. 29 (1970), 201-215. MR 40 \#7692.

9. J. W. Jerome and L. L. Schumaker, Applications of $\varepsilon$-entropy to the computation of n-widths, Proc. Amer. Math. Soc. 22 (1969), 719-722. MR 40 \#614.

Department of Mathematics, Northwestern University, Evanston, Illinois 60201 